\title{
MAGNETIC RESPONSE OF AN ORGANISM AND ITS LUNAR RELATIONSHIPS ${ }^{1}$
}

\author{
F. A. BROWN, JR., H. M. WEBB AND W. J. BRETT \\ Departments of Biological Sciences, Northwestern University, Goucher College and \\ Indiana State Teachers College, and the Marine Biological Laboratory, \\ Woods Hole, Mass.
}

It has been demonstrated (Brown, Brett, Bennett and Barnwell, 1960) that mud-snails, Nassarius obsoleta, initially directed magnetic southward in a symmetrical field constant for all factors normally considered able to influence their orientation, exhibit a daily rhythm in the direction of their mean paths. The amount of dispersion of paths of a population of snails about their mean path also displays a daily rhythm. Both of these characteristics of spatial orientation were shown to be quantitatively alterable, in a manner highly significant statistically, by experimentally changing the strength of the ambient magnetic field. Although much of the observed variation, both in the orientation of control animals in the earth's field and in the modified orientation in response to experimentally increased strengths of the magnetic field, was accounted for in terms of the daily rhythm of response, much variation still remained unaccounted for. The following study was made to determine whether lunar periodisms in responsiveness to the magnetic field were also present.

\section{Methods and Materials}

The apparatus, and methods of obtaining the data, utilized in this study have already been reported in detail elsewhere (Brown, Brett, Bennett and Barnwell, 1960). In essence, measurements were made of the average amount of clockwise or counter-clockwise turning of snails as they traversed a $3-\mathrm{cm}$. course immediately following their emergence from a straight, narrow corridor directed toward the magnetic south into an illuminated, symmetrical, constant field. Each of 564 experimental series, obtained during the period June 28 through August 29, 1959, included two groups of exits of ten snails each in the earth's natural magnetic field, two groups of ten in a magnetic field increased 10 -fold and oriented as the natural one $(\mathrm{N}-\mathrm{S})$, and two groups of ten in a nearly equally strengthened field rotated clockwise through $90^{\circ}$ so that the north-seeking pole of the bar magnet was directed west $(\mathrm{E}-\mathrm{W})$.

Data were obtained for hours of the day from 5 AM through 9 PM, E.S.T., with no hour of this period represented by fewer than nine series, nor more than 50 series, of 60 snail exits.

\section{Lunar-day rhythm of mean path}

\section{Results}

All the data on orientation of the snails for each calendar day were rearranged to assume their proper relationship as approximate hours of lunar days. When

1 This research was aided by a contract between the Office of Naval Research, Department of the Navy, and Northwestern University, \#1228-03. 
more than one series was recorded for any single hour of a given day, these were combined to yield a single average value. The average direction and amount of turning of the snails as a function of hour of the lunar day is indicated in Figure 1 for the control snails, those in the artificially augmented N-S magnetic field and those in the E-W field. Each of the three differently treated groups is dealt with here as a separate sample. Not only is there a clear mean lunar-day cycle of

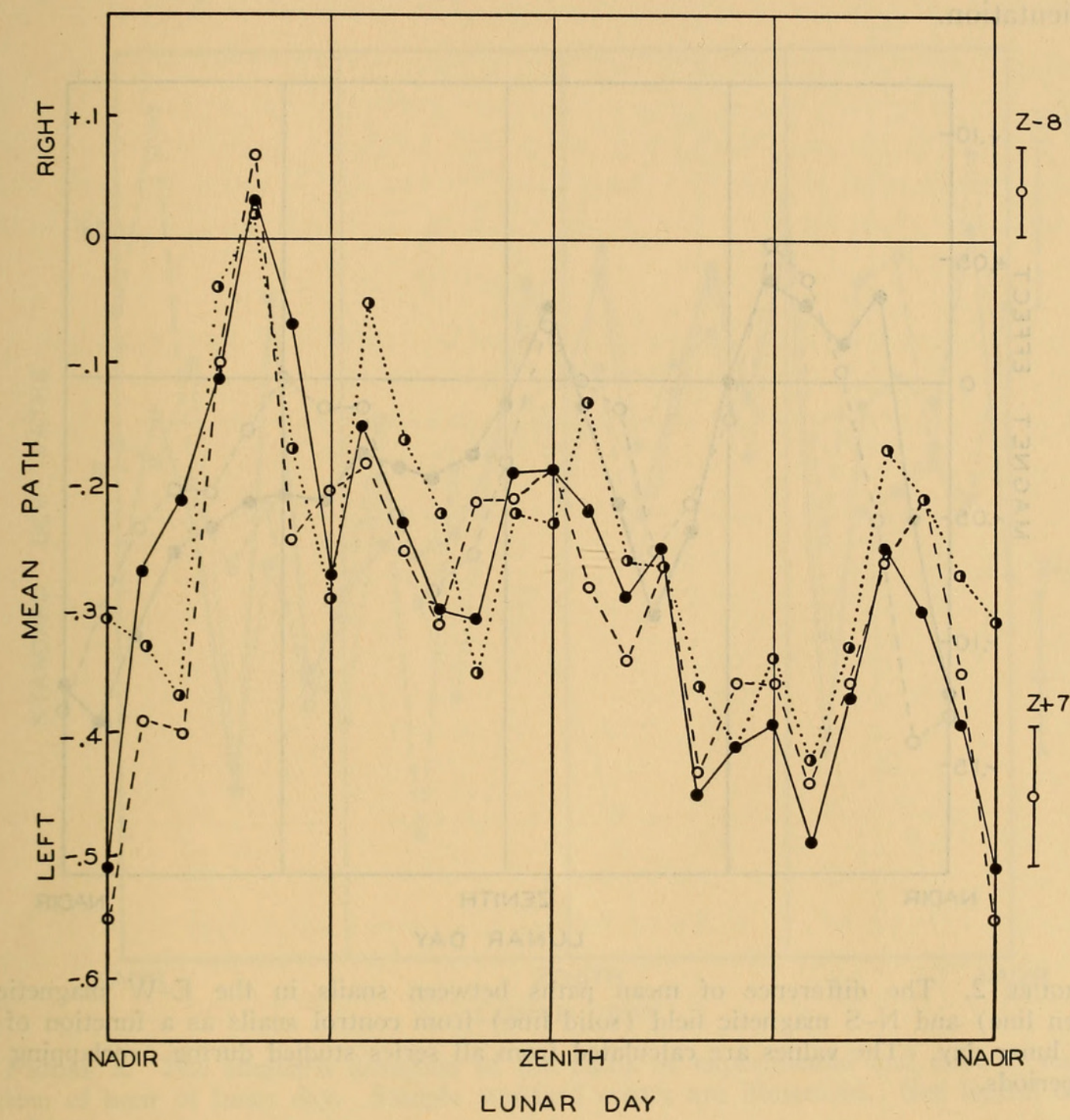

FIGURE 1. The mean paths of snails in the E-W magnetic field (broken line), N-S magnetic field (solid line), and control snails solely in the earth's field (dotted line) as a function of hours of the lunar day. Standard errors of means at two selected times are depicted.

turning, but the three samples exhibit a considerable similarity in the form of the mean cycle despite relatively wide differences between values for the three samples within individual series of 60 (See Brown, Brett, Bennett and Barnwell, 1960).

Not only is the mean lunar-day cycle of turning of essentially the same amplitude as the comparable, previously described solar-day one, but displays a general similarity to it in gross form, though appearing to mirror-image the daily one in 
secondary superimposed fluctuations over the period the moon is above the horizon. As with the solar-day cycle, minimum left-turning or maximum right-turning occurs about the fourth hour of the lunar day or near the time of moon-rise, and maximum left-turning occurs at lunar nadir. Standard errors of the means for the fourth (zenith minus 8) and the nineteenth hours (zenith plus 7 ), calculated from all data for these hours, are indicated on the figure. These values indicate the high degree of statistical significance of this lunar-day fluctuation of mean path of orientation.

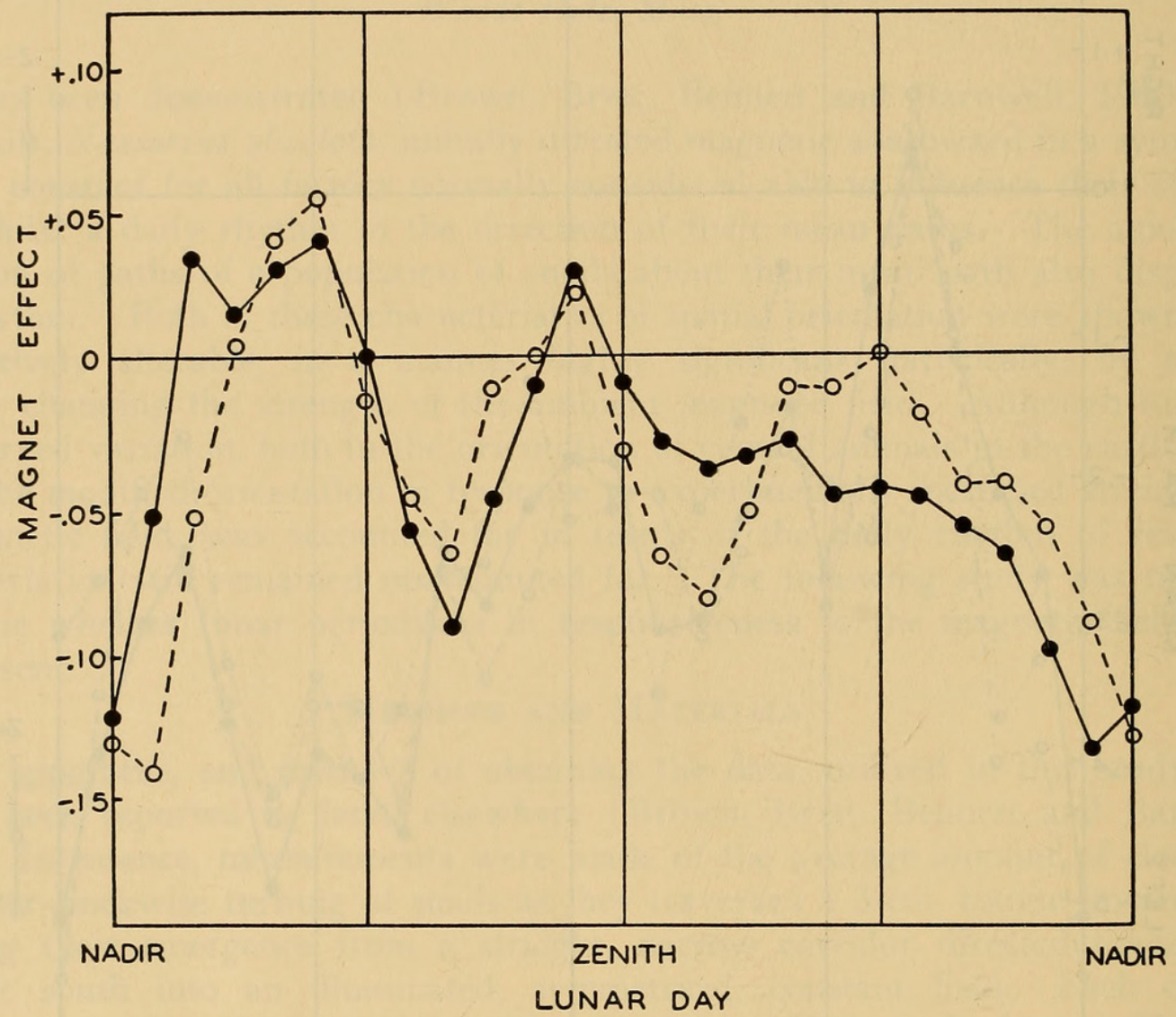

FIGURE 2. The difference of mean paths between snails in the E-W magnetic field (broken line) and N-S magnetic field (solid line) from control snails as a function of hours of the lunar day. The values are calculated from all series studied during overlapping threehour periods.

\section{Lunar rhythm in effect of experimentally augmented magnetic fields}

The average effect of the imposed magnetic fields for overlapping groups of three lunar-day hours were expressed as differences from the comparable three-hour control samples and the mean lunar-day fluctuation in this magnetic effect is plotted in Figure 2. Such three-hour grouping of the data was employed (1) to reduce as far as possible the apparent significance of individual points based upon relatively small numbers of series, and (2) to reduce irregularities in the lunar-day cycle reflecting incomplete randomization of the solar-day cycle as a consequence of the occurrence of occasional solar days lacking in data. As indicated in Figure 2, a maximum right-turning response to the experimental magnetic fields occurs near 
the time of moon-rise, and a maximum left-turning response is seen at lunar nadir. This is true whether one deals with either the $\mathrm{N}-\mathrm{S}$ or $\mathrm{E}-\mathrm{W}$ experimental fields. The lunar-day pattern suggests three progressively decreasing maxima as one moves through the lunar day. The overwhelming preponderance of negative values, indicating the induction of left-turning by the experimental procedure, clearly corroborates the results of the earlier solar-day study in demonstrating this response to the experimentally imposed weak fields.

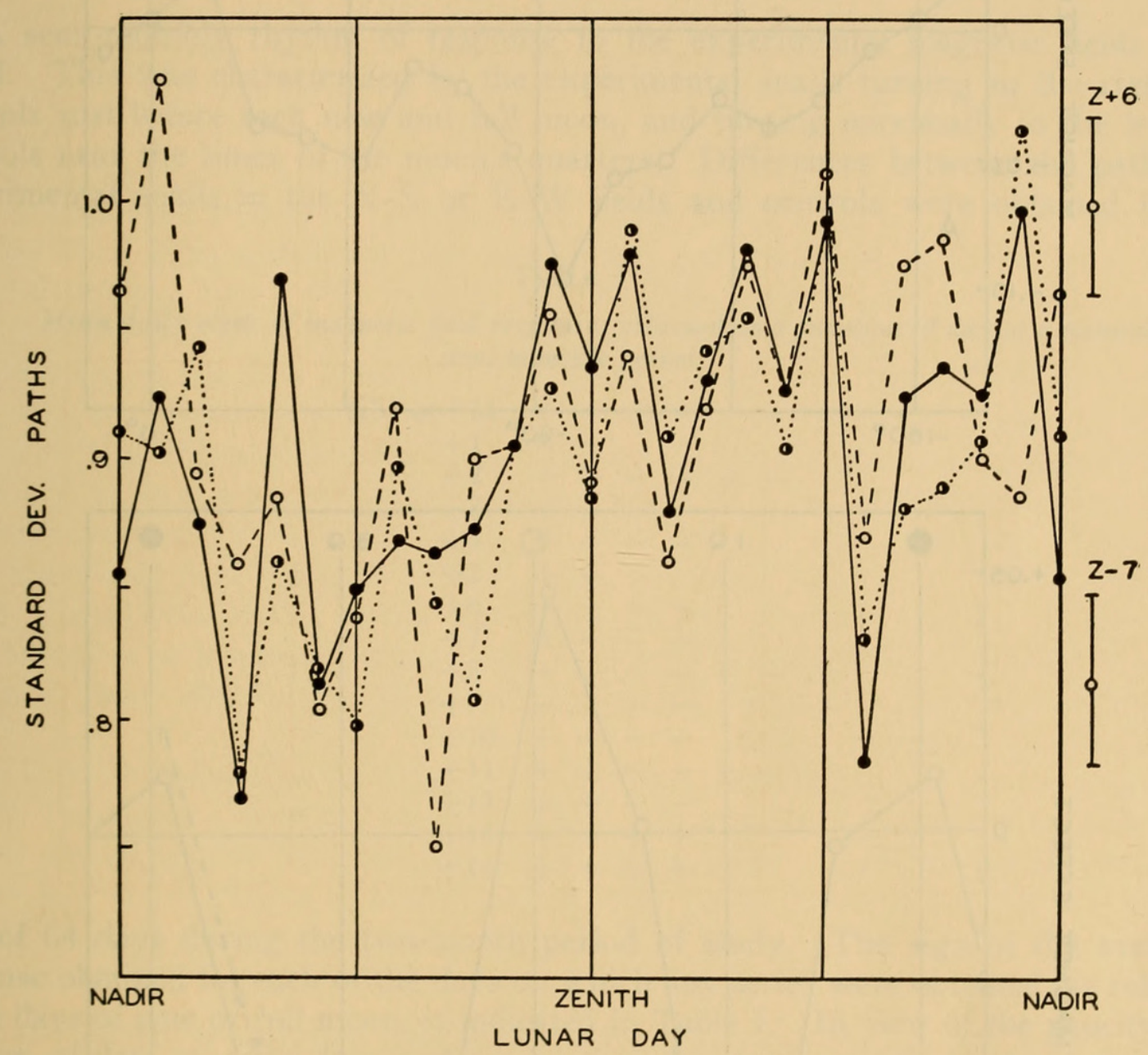

Figure 3. The standard deviation of the paths of experimental and control snails as a function of hour of lunar day. Sample standard errors are illustrated. See legend of Figure 1 for key.

\section{Standard deviation of paths}

In view of the solar-day rhythm of dispersion of paths established previously and indicating both right and left turning responses to the magnetic field, the standard deviations of all the samples of 20 controls, 20 animals in the N-S experimental field and 20 in the E-W field were next rearranged to ascertain whether a lunar-day fluctuation occurred also in this parameter. In Figure 3 is seen the mean lunar-day fluctuation of standard deviations for the month-period, July 6August 4, for each of the two experimental conditions and the controls, separately. Standard errors of the means for the seventh hour before zenith and the sixth hour 

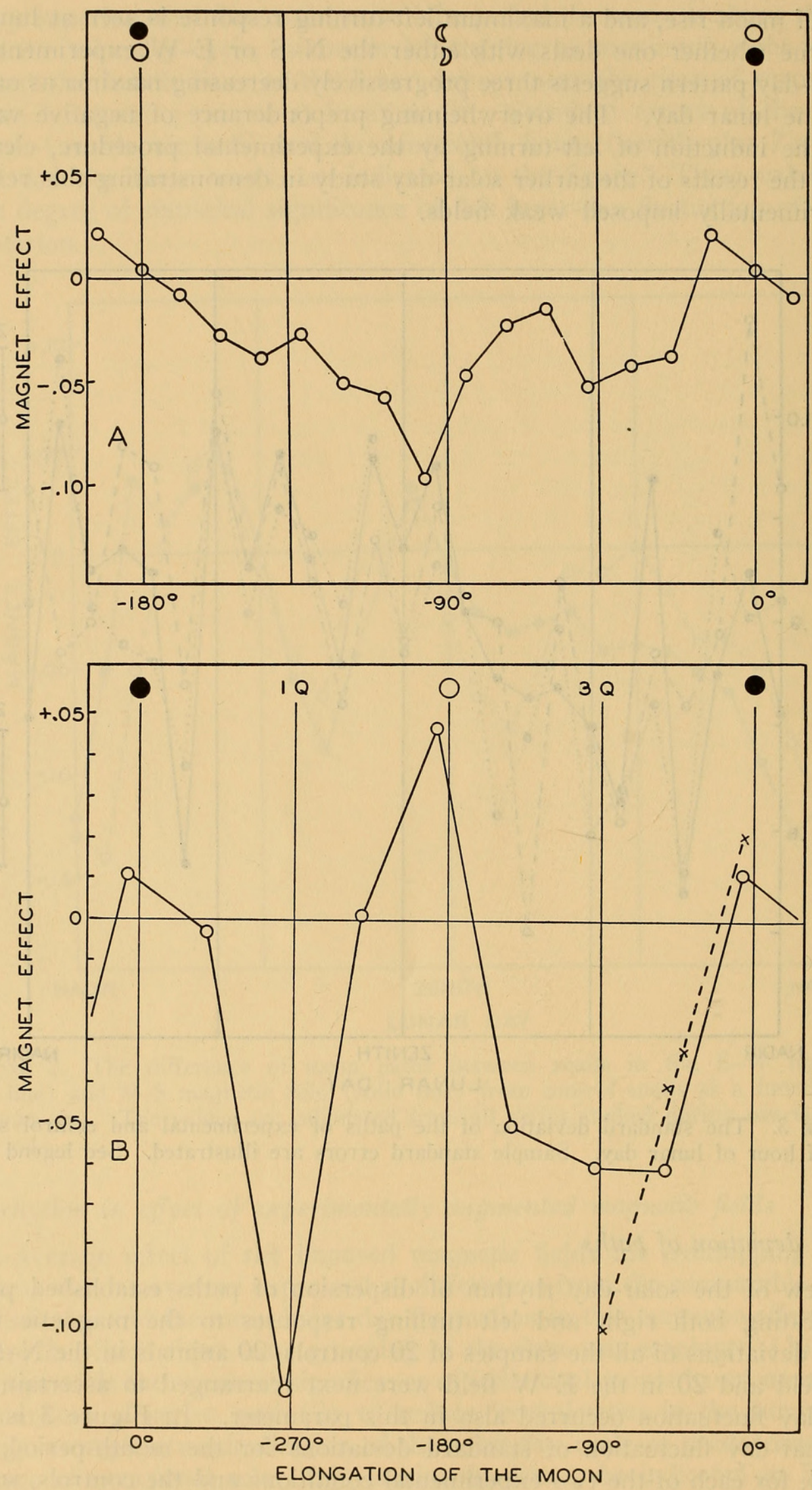

Figure 4. A-B. 
after zenith indicate the highly significant character of this lunar-day cycle. When, instead, all data for the two-month period were used, this cycle was very similar, but had slightly increased amplitude and statistical significance. However, the particular monthly period was selected since the most complete series of daily studies were conducted during this period, and, hence, more complete randomization of the solar-daily cycle was assured.

\section{Semi-monthly cycle in the mean daily sign and amount of turning}

A semi-monthly rhythm of response to the experimental magnetic fields was noted. This was characterized by the experimental snails turning to the right of controls just before each new and full moon, and turning maximally to the left of controls near the times of the moon's quarters. Differences between the paths of experimental snails in the $\mathrm{N}-\mathrm{S}$ or $\mathrm{E}-\mathrm{W}$ fields and controls were obtained for a

\section{TABLE I}

Mean daily signs of magnetic field responses obtained as a function of day of a natural semi-monthly period

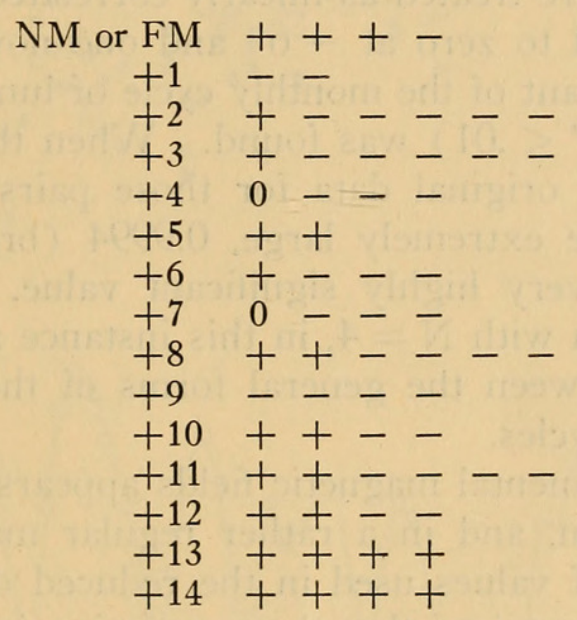

total of 64 days during the two-month period of study. The sign of the average response obtained for each of the days on which any series were obtained, as related to the days of new or full moon, is indicated in Table I. In view of the paucity, or absence, of data on some days and the relative abundance on other days it was felt that a more accurate determination of the mean form of this semi-monthly cycle could be obtained by calculating averages of all available series for overlapping periods of three consecutive days each. The results of such a procedure are plotted in Figure 4A.

The values were obtained by considering each day on a semi-monthly frame of

FIGURE 4. A. Semi-monthly cycle in mean daily difference between paths of experimental and control snails. The mean values from overlapping three-day periods of study comprise the points. B. The solid line indicates the fluctuation through the synodic month of the response to the experimental magnetic field based upon 3- to 5-day grouped data, selected to provide mean values at about $45^{\circ}$ intervals of elongation of the moon relative to the sun. The broken line shows the relationship after averaging the original data for the two semi-monthly periods, considering both the times of new and full moon as $0^{\circ}$, and using the single range $0^{\circ}$ to $90^{\circ}$. 
reference of the scales new moon through new moon minus 14 days and full moon through full moon minus 14 days. This provided an arbitrary 15-day semimonthly period, an adequately close approximation, for the purposes at hand, to the mean, natural 14.8-day one.

In order to assay the statistical significance of this semi-monthly cycle the correlation of the effect of the magnets with the elongation of the moon relative to the sun was assayed. The daily angle of elongation is $12.2^{\circ}$. However, inspection of Figure 4A suggested that the maxima and minima preceded the days of the new and full moon, or its quarters, by about half a day, or by about $-6^{\circ}$. This value, consequently, was treated as the corrected zero one. The data were next combined into eight groups of days in the synodic month: (1) New moon, -1 , and -2 ; (2) $-3,-4,-5,-6$; (3) $-7,-8,-9$; (4) $-10,-11,-12,-13,-14$; (5) Full moon $-1,-2$; (6) $-3,-4,-5,-6$; (7) $-7,-8,-9$; and (8) - 10, $-11,-12,-13,-14$. These times were selected to give values centered on times close to those in which the earth-moon and sun-moon axes were parallel with one another $\left(0^{\circ}\right)$, at right angles to one another $\left(90^{\circ}\right)$ and midway between these two $\left(45^{\circ}\right)$. The eight values are plotted in Figure $4 \mathrm{~B}$, now corrected for the $-6^{\circ}$. When these were treated as linearly correlated with the angular elongation of the moon corrected to zero at $-6^{\circ}$ and one now remained with the 0 to $-90^{\circ}$ range of each quadrant of the monthly cycle of lunar elongation, a coefficient of correlation of $0.8486(P<.01)$ was found. When the same data were further reduced by combining the original data for those pairs of values related to one another by $180^{\circ}$, r became extremely large, 0.9994 (broken line in Figure 4B). This last is obviously a very highly significant value. The substantially lower value of $r$ with $N=8$ than with $N=4$, in this instance appeared to reflect a quite symmetrical difference between the general forms of the pre-new-moon and prefull-moon semi-monthly cycles.

The response to experimental magnetic fields appears definitely correlated with the elongation of the moon, and in a rather regular manner. However, the restrictions in the number of values used in the reduced data clearly do not permit one to conclude with any certainty that the correlation is a truly linear one.

\section{Synodic monthly fluctuation in mean daily standard deviation of paths}

In Figure 5A are plotted together the mean daily standard deviations of paths of the controls, and the experimental snails in the N-S and E-W magnetic fields. Each is treated separately. During the first forty days of the study there appears to be a synodic monthly fluctuation in standard deviation, with the maximum deviation about two days prior to new moon and minimum deviation about the time of full moon. The last 13 days of data are not in any fundamental manner incompatible with the view of a continuing monthly periodism of the same general character, but do contain, over a short period, some tremendous fluctuations in the standard deviations which clearly can not themselves be part of a simple monthly cycle of the relative regularity of the first. This suggests significantly differing responses of the snails of an aperiodic character, in the constant conditions.

Figure $5 \mathrm{~B}$ is a plot of the mean daily barometric pressure values for the corresponding period of time. The barometric pressure changes appear related more 
than coincidentally to the fluctuations in standard deviations of the snail orientation. Not only are the snail orientation and the barometric pressure fluctuations the mirror-images of one another in gross general trends, but when the barometric pressure fluctuation is treated as a two-day lead correlation on snail orientation,

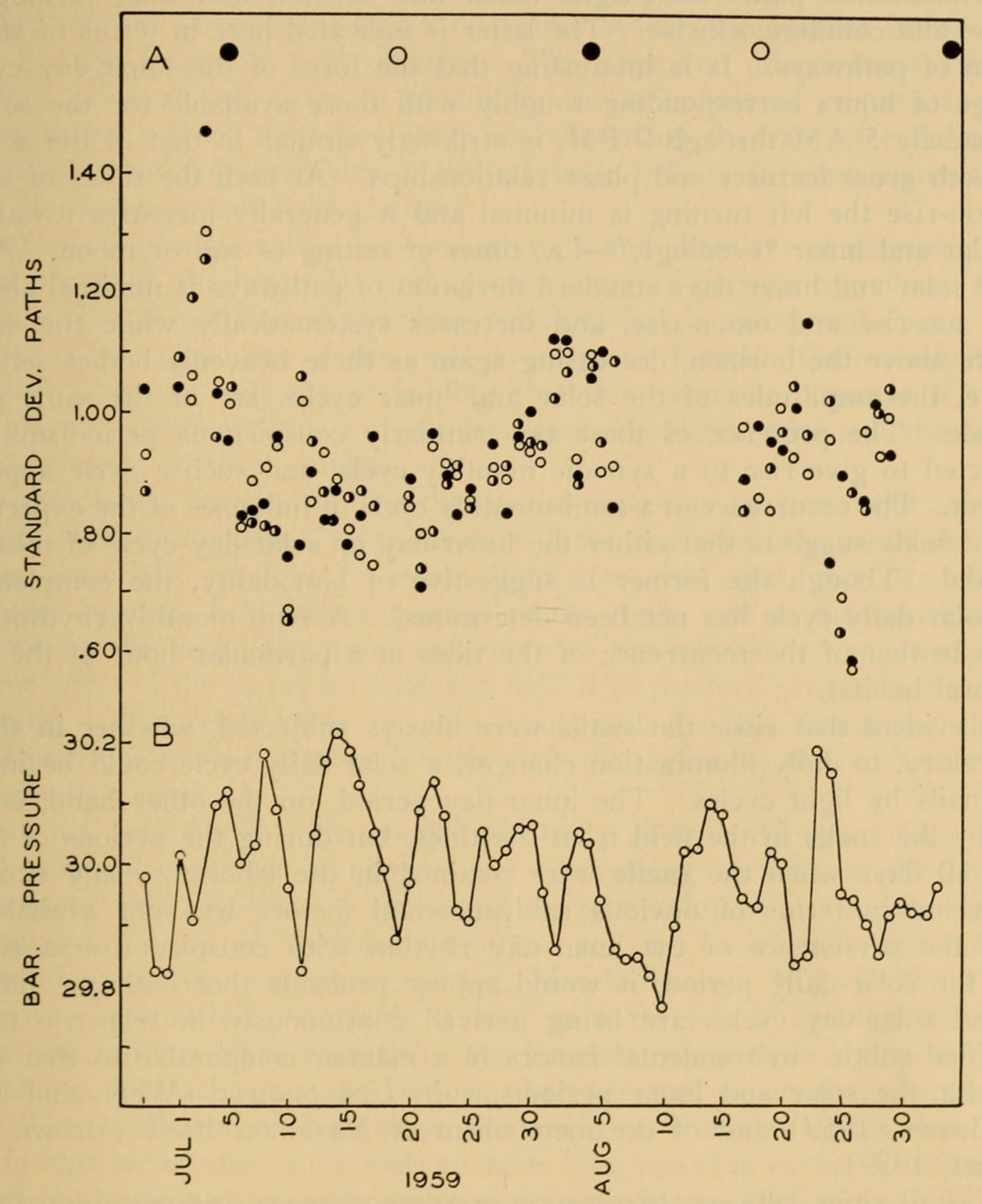

Figure 5. A. The mean daily standard deviations of all snail paths for the whole period of study. Open circles, snails in E-W magnetic field; closed circles, in N-S magnetic field; half open circles, controls. B. Mean daily barometric pressure for the period corresponding to (A).

even a number of the major irregularities in the fluctuations of snail orientations, and particularly those of the last thirteen days, become strikingly accounted for in these terms. The calculated two-day lead correlation was $\mathrm{r}=0.33 \pm 0.08(\mathrm{~N}=$ $141)$, clearly highly significantly different from zero. 


\section{Discussion}

There appears little reason to doubt that spatial orientation of snails, expressed as an amount of turning, or a klinokinesis, possesses a lunar rhythmicality. This is displayed first as lunar-daily, or approximately 24.8-hour, periodicities in both mean orientational paths with signs taken into account and total turning, both clockwise and counterclockwise. The latter is indicated here in terms of standard deviation of pathways. It is interesting that the form of the lunar-day cycle, in the range of hours corresponding roughly with those available for the solar-day study, namely 5 AM through 9 PM, is strikingly similar to that of the solar-day one in both gross features and phase relationships. At both the times of sun-rise and moon-rise the left turning is minimal and it generally increases towards the early solar and lunar "evenings,"-i.e., times of setting of sun or moon. Also, in both the solar and lunar days standard deviation of pathways is minimal about the time of sun-rise and moon-rise, and increases systematically while the sun and moon are above the horizon, decreasing again as these heavenly bodies set. Furthermore, the amplitudes of the solar and lunar cycles are of the same general magnitude. The presence of these two similarly conspicuous periodisms would be expected to give rise to a synodic monthly cycle, and such a cycle appears to be present. The occurrence of a semi-monthly cycle of influence of the experimental magnetic fields suggests that either the lunar-day or solar-day cycle of mean path is bimodal. Though the former is suggestive of bimodality, the complete form of the solar-daily cycle has not been determined. A semi-monthly rhythm would conform to that of the recurrence of the tides at a particular hour of the day in the natural habitat.

It is evident that since the snails were always subjected, whether in the field or laboratory, to daily illumination changes, a solar-daily cycle could be imparted to the snails by light cycles. The lunar-day period, on the other hand, could be gained by the snails in the field from the tides, but during the periods of as long as 8 to 10 days when the snails were retained in the laboratory any exogenous lunar period in terms of obvious environmental factors was not available. In view of the persistence of the lunar-day rhythm with conspicuousness equal to that of the solar-daily period, it would appear probable that both the lunar-day cycle and solar-day cycles are being derived continuously in response to some unidentified subtle environmental factors in a manner comparable to that demonstrated for the solar and lunar periodisms in Uca pugnax (Webb and Brown, 1958; Brown, 1959) and of the metabolism of Nassarius itself (Brown, Webb and Brett, 1959).

The similarities between barometric pressure changes and certain parameters of spatial orientation of snails suggest strongly that orientation in the snails is in some manner dependent upon subtle environmental factors other than magnetic fields and that these other factors may also induce considerable alteration in snail behavior. This is indicated, since even the experimentally augmented magnetic fields may be overriden by the factor correlated with the barometric pressure. One possible factor which has been suggested is electrostatic field (Webb, Brown and Brett, 1959). The correlation with barometric pressure recalls the correlation observed for metabolic fluctuations in the snails in constant conditions, including pressure, during the summer of 1958 (Brown, Webb and Brett, 1959), except 
for its being a mirror-image. Since there is reason to believe (Brown, Brett, Bennett and Barnwell, 1960) that the spatial orientation described in this study is at least in some measure a klinokinetic response to the natural and experimental magnetic fields, it is possible that fluctuations in other subtle geophysical factors can influence the sensitivity of the magnetic responder-system. However, the possibility can not be excluded that a responder system for another factor is being altered by the magnetic field changes. The apparent effectiveness of certain barometric pressure correlates in modifying the orientational responses, and the well-established role of these correlates in influencing the rate of cellular oxidations, suggest that the sensitivity to the magnetic field is in some manner related to rate of oxidative metabolic changes.

These striking relationships between strength and character of magnetic orientation and phases of the lunar-day and synodic monthly periods contribute, together with the earlier demonstrated solar-day relationships, a substantial degree of predictability to the responses of the snail to magnetic forces. Collectively they increase still more not only the probability that this magnetic response is real, but that it plays a significant role in both the lunar-dominated tidal and the solar-day periodic behavior of these animals.

It is interesting, further, to note that in the lunar-day fluctuation in orientation, with sign taken into account, those times of lunar day when the mean path of the snails is essentially straight or even slightly to the right, the experimentally augmented magnetic field effects turning to the right, and as the snails normally orient during the lunar day more and more to the left as one approaches lunar nadir, the effect of the increased magnetic field is to produce progressively stronger left-turning. This relationship is quite comparable to that found for the solar day and provides further support for the view that response to the earth's natural field normally occurs.

There has been noted a remarkable similarity in both the gross form and the detailed trimodal character of progressively decreasing maxima of the lunar-day, $\mathrm{N}-\mathrm{S}$ magnetic field responses of snails and the mirror-image of a highly significant mean lunar-day cycle of spontaneous motor activity found in white mice over the 5-month, partially overlapping period, March through July, 1959 (Terracini and Brown, unpublished) similarly treated as a three-hour moving mean. The maxima and minima were essentially synchronous when the two cycles, studied in two places, Massachusetts and Illinois, were adjusted to simultaneity (universal time). The coefficient of correlation for the two trimodal cycles was -0.85 . Similarly high simultaneous correlations have been reported earlier between fluctuations of the nucleonic component of cosmic radiation in Illinois and fiddler crab and sea-weed metabolism in constant conditions in Massachusetts (Brown, Webb and Bennett, 1958). This similarity between the cycles of mice activity and response of snails to the magnetic field supports the hypothesis that in this magnetic response we are dealing with a widely, probably universally, occurring biological phenomenon.

The proof of a remarkable sensitivity of an organism to one subtle environmental factor (magnetic field) operating at an intensity only slightly above the earth's natural one, and the evidence for its modification in these constant conditions by fluctuations in a second natural factor, poses even the very fundamental problem 
of the dispensability, or indispensability, of the earth's milieu of subtle factors and its natural fluctuations, as a normal ecological consideration in organismic survival and species propagation.

\section{Sum MARY}

1. The direction, and mean amount, of turning in snails initially directed southward into a constant symmetrical, illuminated field displays a lunar-day rhythm with minimum turning about the time of moon-rise, and maximum turning at lunar nadir. There is also a lunar-day cycle of standard deviation of snail pathways, with a minimum about moon-rise and a maximum near moon-set.

2. The response of snails to an experimentally augmented magnetic field also exhibits a lunar-day rhythm with maximum turning to the left at lunar nadir.

3. The specific character of the lunar-day rhythm of the response to the experimental magnetic fields gives further support for the view that magnetic field is normally involved in snail orientation.

4. The mean daily response of snails to experimental magnetic fields, expressed as differences from the response of controls in the earth's natural field, displays a semi-monthly rhythm. Maximum right-turning in response to a magnetic increase of 10 -fold over that of the earth occurs one to two days before new and full moon, and maximum left turning just before the times of the first and third quarters of the moon.

5. There is a synodic monthly fluctuation in mean daily standard deviations of snail paths with maximum deviation about two days before new moon and minimum deviation about the time of full moon.

6. Some suggestive correlations are demonstrated between barometric pressure and the spatial orientation of snails in an environment constant with respect to all generally accepted orienting factors.

7. It is pointed out that similarities in influence of some unidentified barometric pressure correlates on (a) magnetic orientation of snails, (b) general cellular oxidations, and (c) spontaneous activity cycles present reasons for postulating that the latter two phenomena are in some manner related to the magnetic field response and suggest that response to magnetic field is a widely distributed biological phenomenon.

8. Evidence is presented that suggests there is a biological influence of a universal-time-related, rhythmic, environmental factor.

\section{LITERATURE CITED}

Brown, F. A., JR., 1959. Living clocks. Science, 130: 1535-1544.

Brown, F. A., JR., W. J. Brett, M. F. Bennett and F. H. Barnwell, 1960. Magnetic response of an organism and its solar relationships. Biol. Bull., 118: 367-381.

Brown, F. A., Jr., H. M. Webb and M. F. Bennetr, 1958. Comparisons of some fluctuations in cosmic radiation and in organismic activity during 1954, 1955 and 1956. Amer. J. Physiol., 195 : 237-243.

Brown, F. A., JR., H. M. WebB ANd W. J. Brett, 1959. Exogenous timing of solar and lunar periodisms in metabolism of the mud-snail, Ilyanassa (Nassarius) obsoleta, in laboratory constant conditions. Gunma J. Med. Sci., 8: 233-242.

WebB, H. M., AND F. A. Brown, JR., 1958. The repetition of pattern in the respiration of Uca pugnax. Biol. Bull., 115: 303-318.

Webb, H. M., F. A. Brown, Jr. And W. J. Brett, 1959. Effects of imposed electrostatic fields on rate of locomotion in Ilyanassa. Biol. Bull., 117: 430-431. 


\section{$2 \mathrm{BHL}$ Biodiversity Heritage Library}

Brown, Frank A., Webb, H. Marguerite, and Brett, W. J. 1960. "Magnetic response of an organism and its lunar relationships." The Biological bulletin 118, 382-392. https://doi.org/10.2307/1538816.

View This Item Online: https://www.biodiversitylibrary.org/item/110976

DOI: https://doi.org/10.2307/1538816

Permalink: https://www.biodiversitylibrary.org/partpdf/2143

\section{Holding Institution}

Smithsonian Libraries

\section{Sponsored by}

Biodiversity Heritage Library

\section{Copyright \& Reuse}

Copyright Status: In copyright. Digitized with the permission of the rights holder.

License: http://creativecommons.org/licenses/by-nc-sa/3.0/

Rights: https://biodiversitylibrary.org/permissions

This document was created from content at the Biodiversity Heritage Library, the world's largest open access digital library for biodiversity literature and archives. Visit BHL at https://www.biodiversitylibrary.org. 